\title{
Multiple-relay selection in amplify-and-forward cooperative wireless networks with multiple source nodes
}

\author{
Jinsong $\mathrm{Wu}^{1 *}$, Yimin D Zhang ${ }^{2 *}$, Moeness $\mathrm{G} \mathrm{Amin}^{2}$ and Murat Uysal ${ }^{3}$
}

\begin{abstract}
In this article, we propose multiple-relay selection schemes for multiple source nodes in amplify-and-forward wireless relay networks based on the sum capacity maximization criterion. Both optimal and sub-optimal relay selection criteria are discussed, considering that sub-optimal approaches demonstrate advantages in reduced computational complexity. Using semi-definite programming convex optimization, we present computationally efficient algorithms for multiple-source multiple-relay selection (MSMRS) with both fixed number and varied number of relays. Finally, numerical results are provided to illustrate the comparisons between different relay selection criteria. It is demonstrated that optimal varied number MSMRS outperforms optimal fixed number MSMRS under the same power constraints.
\end{abstract}

\section{Introduction}

Multihop relaying has emerged as a promising approach to achieve high-rate coverage in wireless communications $[1,2]$. Several amplify-and-forward (AF) and decode-andforward (DF) relaying techniques have been introduced such as in $[2,3]$. Following those pioneer works, a number of cooperative diversity schemes have been proposed, including, for example, distributed space-time coding [3$5]$, adaptive power control for relay networks or relay beamforming [6-9], and relay selection [10-19].

The objective of relay selection is to achieve higher throughput or lower error probability through choosing one or more relays for transmission according to channel conditions. In comparison to relay beamforming, relay selection is attractive due to its deployment of simpler signaling scheme and energy saving. Most currently available relay selection approaches assume only a single source node [10-12,14-18], and can be classified into two categories:

1. A majority of relay selection rules are restrictive in the sense that they either always use all the available

*Correspondence: wujs@ieee.org; yimin.zhang@villanova.edu

1 Bell Laboratories, Alcatel-Lucent, Shanghai 201206, P.R. China

${ }^{2}$ Center for Advanced Communications, Villanova University, Villanova, PA 19085, USA

Full list of author information is available at the end of the article relays or always use just a single relay, such as in [10-18,20-29]. In [21], four simple relay selection criteria are described: Two criteria are based on the selection of a single relay according to mean channel gains, while the other two select all available relays. Selecting all available relays are the simplest approach with multiple relays, and this approach may not be allowed when the sum power limit is less than the summation of the power values of all available relays. A single-relay node is selected based on average channel state information (CSI), e.g., distance or path loss $[20,22,30]$, and on the instantaneous fading states of the various links such as in [23].

2. Multiple-relay selection for a single source has attracted attention as well [31-33]. Jing and Jafarkhani proposed sub-optimal two-step optimization approaches for single-source multiple-relay selection in $[31,33]$ : In the first step, phase rotation is performed at each relay, and thus only power allocation is considered due to signal-to-noise ratio (SNR) consisting of a summation of purely real terms. In the second step, several sub-optimal methods were introduced [31,33]:

(a) By introducing the idea of relay ordering, several schemes with linear complexity were proposed;

\section{Springer}

(c) 2012 Wu et al: licensee Springer. This is an Open Access article distributed under the terms of the Creative Commons Attribution License (http://creativecommons.org/licenses/by/2.0), which permits unrestricted use, distribution, and reproduction in any medium, provided the original work is properly cited. 
(b) Based on recursion, a scheme with quadratic complexity was proposed.

Although both single- and multiple-relay selection approaches for a single source node network have been investigated, relay selection approaches for multiple source nodes are rarely addressed in literature. Only the following three existing publications [34-36] have discussed multiple-source relay selection (MSRS) approaches. Elzbieta and Raviraj have proposed MSRS for DF relay networks [34]. Xu et al. have presented MSRS approaches in which only a single source is considered as the desired user over each selected relay per transmission while other sources or users are considered as interferers during the transmission [35]. Guo et al. have analyzed MSRS for opportunistic relays, in other words, only a single source is transmitted over each selected relay per transmission [36]. Further, there have been several recent research works on two-way relay selections [37-40].

In this article, we consider AF relay-based cooperative communication systems for simultaneous multiple-source transmission over each selected relay, and more than one relay is allowed per multiple-source transmission. Each relay is assumed to satisfy practical individual short-term power constraints, that is, each relay has two power levels: zero and its maximum power. This assumption has been used for single-source multiple-relay selection in, for example, [31,33]. The main contributions of this article can be listed as:

1. Based on the sum capacity criteria, we derive and propose several multiple-relay selection techniques in AF relay networks with multiple source nodes.

2. Using semi-definite programming optimization, we propose computationally efficient algorithms for multiple-source multiple-relay selection (MSMRS) in the presence of both fixed number and varied number of relays.

The following notations are used: $(\cdot)^{\mathcal{T}}$ denotes matrix transpose, $(\cdot)^{*}$ conjugate, $(\cdot)^{\mathcal{H}}$ matrix conjugate transpose, $\odot$ Hardmard product operator, $[\mathbf{A}]_{a, b}$ the $(a, b)$ th entry (element) of matrix $\mathbf{A}, \operatorname{tr}(\cdot)$ matrix trace operation, $\operatorname{Re}(\cdot)$ real part of the object (matrix or variable), $\operatorname{Im}(\cdot)$ imaginary part of the object (matrix or variable), $\mathrm{E}_{\alpha}(\cdot)$ expectation over random variable or random variable set $\alpha$, diag (a) denotes a square matrix with all-zeros entries except the main diagonal filled with the entries of the vector $\mathbf{a}, \phi$ denotes empty set, and $\mathbf{X} \succeq 0$ denotes that $\mathbf{X}$ is a positive semi-definite matrix.

\section{System model and problem formulation}

Consider a wireless relay network with $M$ source nodes (transmitters), $K$ relay nodes, and one destination node (receiver). Each node is equipped with a single antenna.
Assume no direct channel path between the source nodes and the destination node. The source nodes and the relay nodes are assumed to share the same transmission channel.

Based on two-phase half-duplex AF relay assumption, we consider a multiple-source AF relay selection approach. The period of one two-phase AF relay procedure is defined as one time channel use. During the $t$ th time channel use, the two-phase AF protocol is performed as follows:

1. In the first phase, the $m$ th source node (transmitter) sends source information symbol $x_{m}^{(t)}$ using power $P_{m}^{(S)}$ to the relay nodes, where $m=1, \ldots, M$, $\mathrm{E}\left(\left|x_{m}^{(t)}\right|^{2}\right)=1$. the information symbols $x_{m}^{(t)}$, $m=1, \ldots, M$, are selected randomly from $M$ independent codebooks. It is assumed that $M$ source nodes simultaneously send uncorrelated signal streams $x_{m}^{(t)}, m=1, \ldots, M$, and the corresponding channel symbols are received at relay $k$ at the same time.

2. In the second phase, $L$ relays with indices $\left\{k_{1}, \ldots, k_{L}\right\}$ are selected according to some criteria, which will be elaborated later. Here, $L, 1 \leq L \leq K$, is an integer, which is referred to as "relay selection order" in this article. Then, the $k_{i}$ th relay, $i=1, \ldots, L$, scales its received signal power to unity, and, using power $P_{k_{i}}^{(R)}$, amplifies and forwards it to the receiver.

Note that, in this two-phase AF protocol, multiple source nodes share the same channels. The transmission and reception among the source nodes, the relay nodes and the destination node are assumed to be perfectly synchronized.

In the $t$ th time channel use, the channel from the $m$ th source node (transmitter) to the $k$ th relay is denoted as $h_{m}^{(k, t)}$ and the channel from the $k$ th relay to the receiver is denoted as $g_{k}^{(t)}$. The channels are modeled as frequency non-selective Rayleigh fading, and are assumed to independently vary over different time channel uses. Denote $v_{k}^{(t)}$ as the noise component at the $k$ th relay, $k=1, \ldots, K$, and denote $w^{(t)}$ as the noise component at the destination node, where $v_{k}^{(t)}$ and $w^{(t)}$ are assumed to be independently and identically distributed (i.i.d.) complex Gaussian random variables with zero mean and unit variance.

During the $t$ th time channel use, the received signal at the $k$ th relay is

$$
r_{k}^{(t)}=\sum_{m=1}^{M}\left(\sqrt{P_{m}^{(S)}} h_{m}^{(k, t)} x_{m}^{(t)}\right)+v_{k}^{(t)} .
$$


The corresponding scaling factor for the $k$ th relay is given by

$$
\beta_{k}^{(t)}=\frac{1}{\sqrt{\sum_{m=1}^{M} P_{m}^{(S)}\left|h_{m}^{(k, t)}\right|^{2}+1}}
$$

During the $t$ th time channel use, the received signal at destination is then obtained as

$$
\begin{aligned}
& y^{(t)}=\sum_{k=1}^{K}\left(\alpha_{k}^{(t)} \sqrt{P_{k}^{(R)}} g_{k}^{(t)} \beta_{k}^{(t)} r_{k}^{(t)}\right)+w^{(t)} \\
& =\underbrace{\sum_{k=1}^{K}\left(\alpha_{k}^{(t)} \sqrt{P_{k}^{(R)}} g_{k}^{(t)} \beta_{k}^{(t)} \sum_{m=1}^{M}\left(\sqrt{P_{m}^{(S)}} h_{m}^{(k, t)} x_{m}\right)\right)}_{s^{(t)}} \\
& +\underbrace{\sum_{k=1}^{K}\left(\alpha_{k}^{(t)} \sqrt{P_{k}^{(R)}} g_{k}^{(t)} \beta_{k}^{(t)} v_{k}^{(t)}\right)+w^{(t)}}_{q^{(t)}},
\end{aligned}
$$

where $\alpha_{k}^{(t)}$ is the relay selection factor, whose value is equal to 0 or 1 , depending upon different relay selection algorithms.

In this article, we choose sum capacity per time channel use as the performance measure for relay selection [4143]. The system sum capacity is given by

$$
C^{(t)}=\frac{1}{2} \log _{2}\left(1+\rho^{(t)}\right)
$$

where $\rho^{(t)}$ is the overall system effective SNR, and obtained in our case as

$$
\rho^{(t)}=\mathrm{E}_{\left\{x_{m}^{(t)}, v_{k}^{(t)}, w^{(t)}\right\}}\left(\left|\frac{s^{(t)}}{q^{(t)}}\right|^{2}\right)=\frac{\mathrm{E}_{\left\{x_{m}^{(t)}\right\}}\left|s^{(t)}\right|^{2}}{\mathrm{E}_{\left\{v_{k}^{(t)}, w^{(t)}\right\}}\left|q^{(t)}\right|^{2}}
$$

In the above, $\mathrm{E}_{\left\{x_{m}^{(t)}\right\}}\left(\left|s^{(t)}\right|^{2}\right)$ and $\mathrm{E}_{\left\{v_{k}^{(t)}, w^{(t)}\right\}}\left(\left|q^{(t)}\right|^{2}\right)$ are given by

$$
\mathrm{E}_{\left\{x_{m}^{(t)}\right\}}\left(\left|s^{(t)}\right|^{2}\right)=\sum_{m=1}^{M}\left(P_{m}^{(S)}\left|\sum_{k=1}^{K}\left(\sqrt{P_{k}^{(R)}} \alpha_{k}^{(t)} g_{k}^{(t)} \beta_{k}^{(t)} h_{m}^{(k, t)}\right)\right|^{2}\right)
$$

and

$$
\mathrm{E}_{\left\{v_{k}^{(t)}, w^{(t)}\right\}}\left(\left|q^{(t)}\right|^{2}\right)=\sum_{k=1}^{K}\left(P_{k}^{(R)}\left|\alpha_{k}^{(t)} g_{k}^{(t)} \beta_{k}^{(t)}\right|^{2}\right)+1
$$

Inserting (6) and (7) into (5), we have

$$
\rho^{(t)}=\frac{\sum_{m=1}^{M}\left(P_{m}^{(S)}\left|\sum_{k=1}^{K}\left(\sqrt{P_{k}^{(R)}} \alpha_{k}^{(t)} g_{k}^{(t)} \beta_{k}^{(t)} h_{m}^{(k, t)}\right)\right|^{2}\right)}{\sum_{k=1}^{K}\left(P_{k}^{(R)}\left|\alpha_{k}^{(t)} g_{k}^{(t)} \beta_{k}^{(t)}\right|^{2}\right)+1} .
$$

Relay selection could be expressed using set partition. Define relay index set $\Omega=\{1, \ldots, K\}$. There exist $L$ distinct relay indices $\left\{k_{1}, \ldots, k_{L}\right\}$, where $1 \leq$ $\left\{k_{1}, \ldots, k_{L}\right\} \leq K$, such that the following hold: $\left\{\alpha_{k}^{(t)}=0, k \notin\left\{k_{1}, \ldots, k_{L}\right\}, 1 \leq k \leq K\right\}$ and $\alpha_{k_{1}}^{(t)}=\cdots=$ $\alpha_{k_{L}}^{(t)}=1$. The optimization problem can be now formulated as $\arg \max \left\{C^{(t)}\right\}$. Since $\log _{a}(\cdot), a>1$, is a monotonous function, the problem is equivalent to $\arg \max \left\{\rho^{(t)}\right\}$.

$\Omega$

Relay selection can be implemented at the destination node (receiver). In this case, the receiver is assumed to know all instantaneous channel state information for source-relay paths and relay-destination paths, which may be obtained through channel estimation. After one relay selection algorithm is performed at the destination node, $\alpha_{k_{1}}^{(t)}=\cdots=\alpha_{k_{L}}^{(t)}$ are obtained, and then the destination node feedbacks one-digit relay selection information to each relay node. The superscripts ${ }^{(t)}$ used in this section will be omitted in the rest of the article to simplify the notations whenever no ambiguity arises.

\section{Fixed number multiple-source multiple-relay selection}

When individual relay power constraints are equal or close, the number of relays may be used as a constraint to stand for sum relay power constraints. In this section, for simultaneous transmission of multiple source signals, the number of relays to be selected is assumed to be a fixed number $L$, where $L>1$. This class of approaches are referred to as fixed number multiple-source multiplerelay selection (FN-MSMRS), and the corresponding set partition of relay indices for relay selection can be defined as

$$
\Omega_{M}^{(F)}=\left\{\Omega \mid\left\{\begin{array}{l}
L>1, \\
L \text { is a fixed positive integer }
\end{array}\right\}\right\} .
$$




\section{Optimal FN-MSMRS}

Using (8) and the values of $\left\{\alpha_{k}^{t}\right\}$, the output SNR of optimal FN-MSMRS (OFN-MSMRS) can be derived as

$$
\rho=\frac{\sum_{m=1}^{M}\left(P_{m}^{(S)}\left|\sum_{d=1}^{L}\left(\sqrt{P_{k_{d}}^{(R)}} g_{k_{d}} \beta_{k_{d}} h_{m}^{\left(k_{d}\right)}\right)\right|^{2}\right)}{\left(\sum_{d=1}^{L}\left(P_{k_{d}}^{(R)}\left|g_{k_{d}}\right|^{2}\left(\beta_{k_{d}}\right)^{2}\right)\right)+1} .
$$

Thus, the proposed selection criterion is

$$
\underset{\Omega_{M}^{(F)}}{\arg \max }\{\rho\} .
$$

\section{Fixed number MSMRS based on semi-definite programming optimization}

The complexity of OFN-MSMRS may be prohibitive particularly when the dimension of the problem becomes larger. Closed-form optimization solutions are unfortunately not possible for (10) due to the involvement of multiple sources. Based on semi-definite programming [44], we propose an efficient approach for FN-MSMRS. First, note that (8) can be written in a matrix form as

$$
\rho=\frac{\mathbf{p}^{\mathcal{T}} \mathbf{P}^{1 / 2} \mathbf{A}_{s} \mathbf{P}^{1 / 2} \mathbf{p}}{\mathbf{p}^{\mathcal{T}} \mathbf{P}^{1 / 2} \mathbf{A}_{n} \mathbf{P}^{1 / 2} \mathbf{p}+1}
$$

where $\mathbf{p}=\left[\alpha_{1}, \ldots, \alpha_{K}\right]^{\mathcal{T}}, \mathbf{P}=\operatorname{diag}\left(P_{1}^{(R)}, \ldots, P_{K}^{(R)}\right), \mathbf{A}_{s}=$ $\sum_{m=1}^{M}\left(P_{m}^{(S)}\left(\mathbf{a}_{s}^{(m)}\right)^{*}\left(\mathbf{a}_{s}^{(m)}\right)^{\mathcal{T}}\right), \mathbf{A}_{n}=\operatorname{diag}\left(\left(\mathbf{a}_{n}\right)^{*} \odot \mathbf{a}_{n}\right)$, $\mathbf{a}_{s}^{(m)}=\left[\beta_{1} h_{m}^{(1)} g_{1}, \ldots, \beta_{K} h_{m}^{(K)} g_{K}\right]^{\mathcal{T}}$, and $\mathbf{a}_{n}^{(m)}=\left[\beta_{1} g_{1}\right.$, $\left.\ldots, \beta_{K} g_{K}\right]^{\mathcal{T}}$. Further, note that $\mathbf{p}$ is a real integer vector with $\{0,1\}$ entries, $\mathbf{A}_{s}$ is a Hermitian matrix, and $\mathbf{A}_{n}$ is a real-valued matrix. It can be readily checked from (6) that

$$
\operatorname{Im}\left\{\mathrm{E}_{\left\{x_{m}\right\}}|s|^{2}\right\}=\operatorname{Im}\left\{\mathbf{p}^{\mathcal{T}} \mathbf{P}^{1 / 2} \mathbf{A}_{s} \mathbf{P}^{1 / 2} \mathbf{p}\right\}=0 .
$$

Thus, (11) can be further simplified in a real-valued matrix form as

$$
\rho=\frac{\mathbf{p}^{\mathcal{T}} \mathbf{P}^{1 / 2} \operatorname{Re}\left\{\mathbf{A}_{s}\right\} \mathbf{P}^{1 / 2} \mathbf{p}}{\mathbf{p}^{\mathcal{T}} \mathbf{P}^{1 / 2} \mathbf{A}_{n} \mathbf{P}^{1 / 2} \mathbf{p}+1} .
$$

Denote

$$
\underline{\mathbf{c}}=2 \mathbf{p}-\mathbf{1}_{K},
$$

where $\mathbf{c}$ is an integer vector with $\{1,-1\}$ entries, and $\mathbf{1}_{K}$ is an all-one column vector of length $K$. For an arbitrary matrix $\mathbf{M}$ of size $K \times K$, the following relationship always holds,

$$
\mathbf{p}^{\mathcal{T}} \mathbf{M p}=\mathbf{c}^{\mathcal{T}} \underline{\mathbf{M c}},
$$

where

$$
\mathbf{c}=\left[1, \underline{\mathbf{c}}^{\mathcal{T}}\right]^{\mathcal{T}},
$$

and matrix $\underline{\mathbf{M}}$ is related to $\mathbf{M}$ by a function $f$ defined as

$$
\underline{\mathbf{M}}=f(\mathbf{M})=\frac{1}{4}\left[\begin{array}{cc}
\left(\mathbf{1}_{K}\right)^{\mathcal{T}} \mathbf{M} \mathbf{1}_{K}\left(\mathbf{1}_{K}\right)^{\mathcal{T}} \mathbf{M} \\
\mathbf{M} \mathbf{1}_{K} & \mathbf{M}
\end{array}\right] .
$$

Now (11) can be re-written as

$$
\rho=\frac{\mathbf{c}^{\mathcal{T}} \mathbf{S c}}{\mathbf{c}^{\mathcal{T}} \mathbf{N c}+1},
$$

where $\mathbf{S}=f\left(\mathbf{P}^{1 / 2} \operatorname{Re}\left\{\mathbf{A}_{s}\right\} \mathbf{P}^{1 / 2}\right)$ and $\mathbf{N}=f\left(\mathbf{P}^{1 / 2}\right.$ $\left.\mathbf{A}_{n} \mathbf{P}^{1 / 2}\right)$.

The optimization problem can be now formulated as

maximize $\rho$,

$$
\text { subject to } \rho=\frac{\mathbf{c}^{\mathcal{T}} \mathbf{S c}}{\mathbf{c}^{\mathcal{T}} \mathbf{N c}+1}
$$

$$
\mathbf{c}^{\mathcal{T}} f\left(\mathbf{I}_{K}\right) \mathbf{c}=L,
$$

$$
\mathbf{c}^{\mathcal{T}} \mathbf{G}_{k} \mathbf{c}=1, k=1, \ldots, K,
$$

where $\mathbf{G}_{k}, k=1, \ldots, K$, are all-zero matrices except $\left[\mathbf{G}_{k}\right]_{k, k}=1, k=1, \ldots, K$. The fixed relay selection order is quantified in (18c). Note that, it is necessary to include individual relay selection factor constraints, such as (18d), which are actually related to individual relay power constraints, otherwise individual relay selection factors can be arbitrary in the optimization process. Only using vector $\mathbf{p}$, it is hard to quantify individual relay selection factor constraints. However, based on the vector transformation in (13) and (15), individual relay selection factor constraints can be advantageously written as the form shown in (18d).

Denote $\mathbf{B}=\mathbf{c c}^{\mathcal{T}}$. Note that $\mathbf{c}^{\mathcal{T}} \mathbf{X} \mathbf{c}=\operatorname{tr}\left(\mathbf{X} \mathbf{c c}^{\mathcal{T}}\right)=$ $\operatorname{tr}(\mathbf{X B})$. Thus, the optimization problem (18) now becomes

maximize $\rho$,

subject to:

$$
\begin{aligned}
& \rho=\frac{\operatorname{tr}(\mathbf{S B})}{\operatorname{tr}(\mathbf{N B})+1}, \\
& \operatorname{tr}\left(f\left(\mathbf{I}_{K}\right) \mathbf{B}\right)=L,
\end{aligned}
$$




$$
\begin{aligned}
& \operatorname{tr}\left(\mathbf{G}_{k} \mathbf{B}\right)=1, k=1, \ldots, K, \\
& \operatorname{rank}(\mathbf{B})=1, \\
& \mathbf{B} \succeq 0 .
\end{aligned}
$$

The optimization problem is not convex due to rank constraint (19e) and fractional constraint (19b). We can perform semi-definite relaxation through removing rank constraint (19e) [45]. Choosing a positive variable $u$, where $\frac{\operatorname{tr}(\mathbf{S B})}{\operatorname{tr}(\mathbf{N B})+1} \geq u$, the above optimization problem can be written as

maximize $u$,

$$
\begin{aligned}
& \text { subject to } \operatorname{tr}\left(\frac{1}{u}(\mathbf{S}-u \mathbf{N}) \mathbf{B}\right) \geq 1, \\
& \operatorname{tr}\left(f\left(\mathbf{I}_{K}\right) \mathbf{B}\right)=L, \\
& \operatorname{tr}\left(\mathbf{G}_{k} \mathbf{B}\right)=1, k=1, \ldots, K, \\
& \mathbf{B} \succeq 0 .
\end{aligned}
$$

The optimization problem (20) is still non-convex. However, using the bisection Algorithm 1 as shown in Appendix, with the aids of convex programming tools, such as CVX $[46,47]$ which we have used in the simulations, the problem (20) can be solved iteratively, since it is quasi-convex in each loop within the bisection Algorithm 1 , where $u$ acts as a constant. This problem (20) can now be efficiently solved by standard interior point algorithms based on semi-definite programming (SDP) [48]. Denote the optimal estimation of $\mathbf{B}$ through the proposed bisection Algorithm 1 as $\widehat{\mathbf{B}}$.

The above SDP procedure requires bisection Algorithm 1, which might introduce higher complexity when the number of iterative loops is high for convergence. Now we may consider another approach without the requirement of an bisection algorithm. Denote

$$
\mathbf{U}=\mathbf{N}+\frac{1}{L} f\left(\mathbf{I}_{K}\right)
$$

Using (20c), we have

$$
\operatorname{tr}(\mathbf{U B})=\operatorname{tr}(\mathbf{N B})+1 .
$$

Denote

$$
\mathbf{w}=\sqrt{\lambda \mathbf{c}}
$$

where $\lambda>0$ is chosen to make sure

$$
\mathbf{w}^{\mathcal{T}} \mathbf{U w}=1
$$

Denote $\mathbf{W}=\mathbf{w w}^{\mathcal{T}}$, and thus

$$
\rho=\frac{\operatorname{tr}(\mathbf{S B})}{\operatorname{tr}(\mathbf{U B})}=\frac{\operatorname{tr}(\mathbf{S W})}{\operatorname{tr}(\mathbf{U W})} .
$$

Through removing rank constraint (19e), the problem (19) is now relaxed to

$$
\begin{aligned}
& \text { maximize } \operatorname{tr}(\mathbf{S W}), \\
& \text { subject to } \operatorname{tr}(\mathbf{U W})=1, \\
& \operatorname{tr}\left(f\left(\mathbf{I}_{K}\right) \mathbf{W}\right)=L \lambda, \\
& \operatorname{tr}\left(\mathbf{G}_{k} \mathbf{W}\right)=\lambda, k=1, \ldots, K, \\
& \mathbf{D} \succeq 0, \\
& \lambda \geq 0 .
\end{aligned}
$$

The problem (26) now could be solved using semidefinite programming without the requirement of a bisection algorithm. Note that the above method could be considered as the extension of Charnes-Cooper algorithm [49] from linear fractional programming to linear quadratic programming.

Note that the above solutions are obtained through removing the rank-1 constraint (19e), which may lead to an increased problem dimension. Thus it is required to convert the semi-definite relaxation solution to some Boolean solution. In $[45,50,51]$, a randomization method has been introduced to achieve this conversion. Note that in those works, the randomization approach is implemented without additional constraint. Here, we extend such randomization approach to support extra constraints, such as (20c). Based on the randomization procedure as proposed in the Appendix, the decision of $\underline{\mathbf{c}}, \widehat{\widehat{\mathbf{c}}}$, can be obtained, where $\widehat{\widehat{\mathbf{c}}}=\left[[\widehat{\mathbf{c}}]_{1,2}, \ldots,[\widehat{\mathbf{c}}]_{1, K+1}\right]^{\mathcal{T}}$ and $[\widehat{\mathbf{c}}]_{1, k}$ is the $k$ th entry of $\widehat{\mathbf{c}}$. It should be noted that Steps 9) and 10) of Algorithm 2 are introduced to satisfy constraint (20c). In $[45,50,51]$, only $\mathbf{c}=\operatorname{sign}\left(\mathbf{V}^{\mathcal{T}} \mathbf{u}\right)$ is used in the randomization process. However, it has been further proved that $\pm \operatorname{sign}(\mathbf{c})=\operatorname{sign}\left(\mathbf{V}^{\mathcal{T}} \mathbf{u}\right)$ holds with probability 1 in Property 2 of [45] $]^{\mathrm{a}}$. Thus it may be meaningful to perform both "+" and "-" of "sign" operations in the randomization process as we have proposed in Steps 9) and 10) of Algorithm 2.

The above proposed MSMRS based on semi-definite programming is termed as SDPFN-MSMRS:

1. In the case of solving Problem 20 and using randomization procedure Algorithm 2: SDPFN-MSMRS B1,

2. In the case of solving Problem 20 and using randomization procedure Algorithm 2 without step 10: SDPFN-MSMRS A1,

3. In the case of solving Problem 26 and using randomization procedure Algorithm 2: SDPFN-MSMRS B2, 
4. In the case of solving Problem 26 and using randomization procedure Algorithm 2 without step 10: SDPFN-MSMRS A2.

Note that both the solutions of SDPFN-MSMRS B1 and SDPFN-MSMRS A1 require bisection algorithms to solve SDP problems iteratively, while both the solutions of SDPFN-MSMRS B2 and SDPFN-MSMRS A2 do not.

\section{Best worse FN-MSMRS and random FN-MSMRS}

Note that (9) cannot be further simplified without additional approximations. Intuitively, it can be questioned whether best-worse single source single-relay selection [33] can be extended to this case. To address this concern, calculate all

$$
a_{k}=\min \left\{\sum_{m=1}^{M}\left(P_{m}^{(S)}\left|h_{m}^{\left(k_{a}\right)}\right|^{2}\right), P_{k}^{(R)}\left|g_{k_{a}}\right|^{2}\left(\beta_{k_{a}}\right)^{2}\right\},
$$

where $k=1, \ldots, K$. Then, permutate $a_{k}$ in descending order such that $a_{\sigma(1)} \geq \cdots \geq a_{\sigma(K)}$, where $\sigma(\cdot)$ denotes the permutation function. This yields $\{\sigma(1), \ldots, \sigma(L)\}$, and such a selection criterion is termed as best worse FN MSMRS (BWFN-MSMRS).

For comparison purpose, we also define random fixed number MSMRS (RANDFN-MSMRS), which randomly selects $L$ relays, as a baseline benchmark FN-MSMRS scheme.

\section{Varied number multiple-source multiple-relay selection (MSMRS)}

When individual relay power constraints are diverse, sum power constraints can no longer be described using a fixed number of relays. Unlike the previous section, we assume in this section that the number of relays to be selected is not predetermined but rather a varied number which is optimized depending on both individual and sum relay power constraints. This class of proposed approaches are abbreviated as VN-MSMRS, and the corresponding set partition of relay indices for relay selection can be defined as

$$
\Omega_{M}^{(V)}=\left\{\Omega \mid\left\{\begin{array}{l}
1 \leq L \leq K \\
L \text { is a integer variable } \\
\sum_{a=1}^{L} P_{k_{a}}^{(R)} \leq P^{(\text {Sum })}
\end{array}\right\} .\right.
$$

For comparison purpose, a baseline benchmark VNMSMRS scheme using predetermined relay selection, PVN-MSMRS, is also defined. In this scheme, a feasible relay selection is chosen, assuming that this selection satisfies given relay power constraints, and no more relays can be added, otherwise the given sum power constraint is violated.

\section{Optimal VN-MSMRS (OVN-MSMRS)}

For VN-MSMRS, the overall effective system SNR is still given by (9). However, $L$ is no longer a fixed number but a variable to be chosen from a set $L \in\{1, \ldots, K\}$. The proposed optimal selection criterion, OVN-MSMRS, becomes

$$
\underset{\Omega_{M}^{(V)}}{\arg \max }\{\rho\} .
$$

\section{Varied number MSMRS based on semi-definite programming optimization}

For VN-MSMRS, the formulation of optimization problem is the same as (18) except that (18c) is replaced by a sum power constraint

$$
\mathbf{c}^{\mathcal{T}} f(\mathbf{P}) \mathbf{c} \leq P^{\text {(Sum) }} .
$$

The corresponding semi-definite relaxation formulation is written as

maximize $u$,

$$
\begin{aligned}
& \text { subject to } \operatorname{tr}\left(\frac{1}{u}(\mathbf{S}-u \mathbf{N}) \mathbf{B}\right) \geq 1, \\
& \operatorname{tr}(f(\mathbf{P}) \mathbf{B}) \leq P^{\text {(Sum) }}, \\
& \operatorname{tr}\left(\mathbf{G}_{k} \mathbf{B}\right)=1, k=1, \ldots, K, \\
& \mathbf{B} \succeq 0
\end{aligned}
$$

The optimization problem (29) can be solved using the bisection procedure similar to the proposed Algorithm 1 as depicted in Appendix. The difference is that Step 4) of the bisection procedure for VN-MSMRS is changed into "solve the SDP optimization problem (29)." To obtain the estimation of $\underline{\mathbf{c}}, \widehat{\widehat{\mathbf{c}}}$, the randomization procedure Algorithm 3 for VN-MSMRS is proposed in Appendix.

The above proposed MSMRS based on semi-definite programming is defined as SDPVN-MSMRS: the SDPVNMSMRS using randomization procedure Algorithm 3 is termed as SDPVN-MSMRS B, while the SDPVN-MSMRS using randomization procedure Algorithm 3 without steps 9) and 10) is called as SDPVN-MSMRS A.

\section{Numerical results}

In this section, we present the performance of the sum capacity per time channel use for the relay selection approaches under considerations. In all figures, the horizontal axis indicates unit power $P$, and $P_{k}^{(R)}, k=1, \ldots, K$, and $P_{m}^{(S)}, m=1 \ldots, M$, are scaled values of $P$. In this section, the number of sources is set to $M=2$. We further assume that channels $h_{m}^{(k, t)}$ and $g_{k}^{(t)}, m=1, \ldots, M$ and $k=$ $1, \ldots, K$, are Rayleigh fading channel gains (modeled as complex Gaussian with zero mean and unit variance), and 
they change independently over different time channel uses.

\section{FN-MSMRS results}

In Figures 1 and 2 , we assume $K=8, L=4$, $P_{k}^{(R)}=P M, k=1, \ldots, K, \quad$ and $\quad P_{m}^{(S)}=P, m=1, \ldots, M$. The settings of randomization procedure in SDPFNMSMRS A1, SDPFN-MSMRS A2, SDPFN-MSMRS B1, and SDPFN-MSMRS B2 are $N_{c}=2$ and $N_{l}=14$.

In Figure 1, we observe that, to achieve the same average sum capacity per time channel use,

1. SDPFN-MSMRS A1, SDPFN-MSMRS A2, SDPFN-MSMRS B1, and SDPFN-MSMRS B2 use less unit power $P$ than BWFN-MSMRS by 1.6 and $1.3 \mathrm{~dB}$, respectively;

2. BWFN-MSMRS use less unit power $P$ than RANDFN-MSMRS by only $2.2 \mathrm{~dB}$;

3. With the advantage of lower complexity, SDPFNMSMRS A1, SDPFN-MSMRS A2, SDPFN-MSMRS B1, and SDPFN-MSMRS B2 require more unit power $P$ than OFN-MSMRS by 2.2 and $2.5 \mathrm{~dB}$, respectively.

It is observed that both SDPFN-MSMRS B1 and SDPFN-MSMRS B2 achieve notably higher average sum capacity over both SDPFN-MSMRS A1 and SDPFNMSMRS A2 for the same unit power P. This also verifies the importance of step 10 of Algorithm 2. With very close performance to SDPFN-MSMRS A1 and SDPFN-MSMRS B1, respectively, SDPFN-MSMRS A2 and SDPFN-MSMRS B2 are quite computationally effective due to avoiding the needs of additional bisection algorithms.

\section{VN-MSMRS results}

In this section, the settings of randomization procedure in SDPVN-MSMRS B and SDPVNMSMRS A are $N_{c}=2$ and $N_{l}=14$.

In Figures 3 and 4 , we assume $K=8, M=2, P^{\text {(Sum) }}=$ $4 P M, \quad P_{k}^{(R)}=P M, k=1, \ldots, K, \quad P_{m}^{(S)}=P, m=1, \ldots, M$. From Figure 3, we observe that, to achieve the same average sum capacity per time channel use,

1. SDPVN-MSMRS B and SDPVN-MSMRS A use less unit power $P$ than PVN-MSMRS by 4.2 and $3.9 \mathrm{~dB}$, respectively,

2. With the advantage of lower complexity, SDPVN-MSMRS B and SDPVN-MSMRS A require more unit power $P$ than OVN-MSMRS by 1.4 and $1.7 \mathrm{~dB}$, respectively.

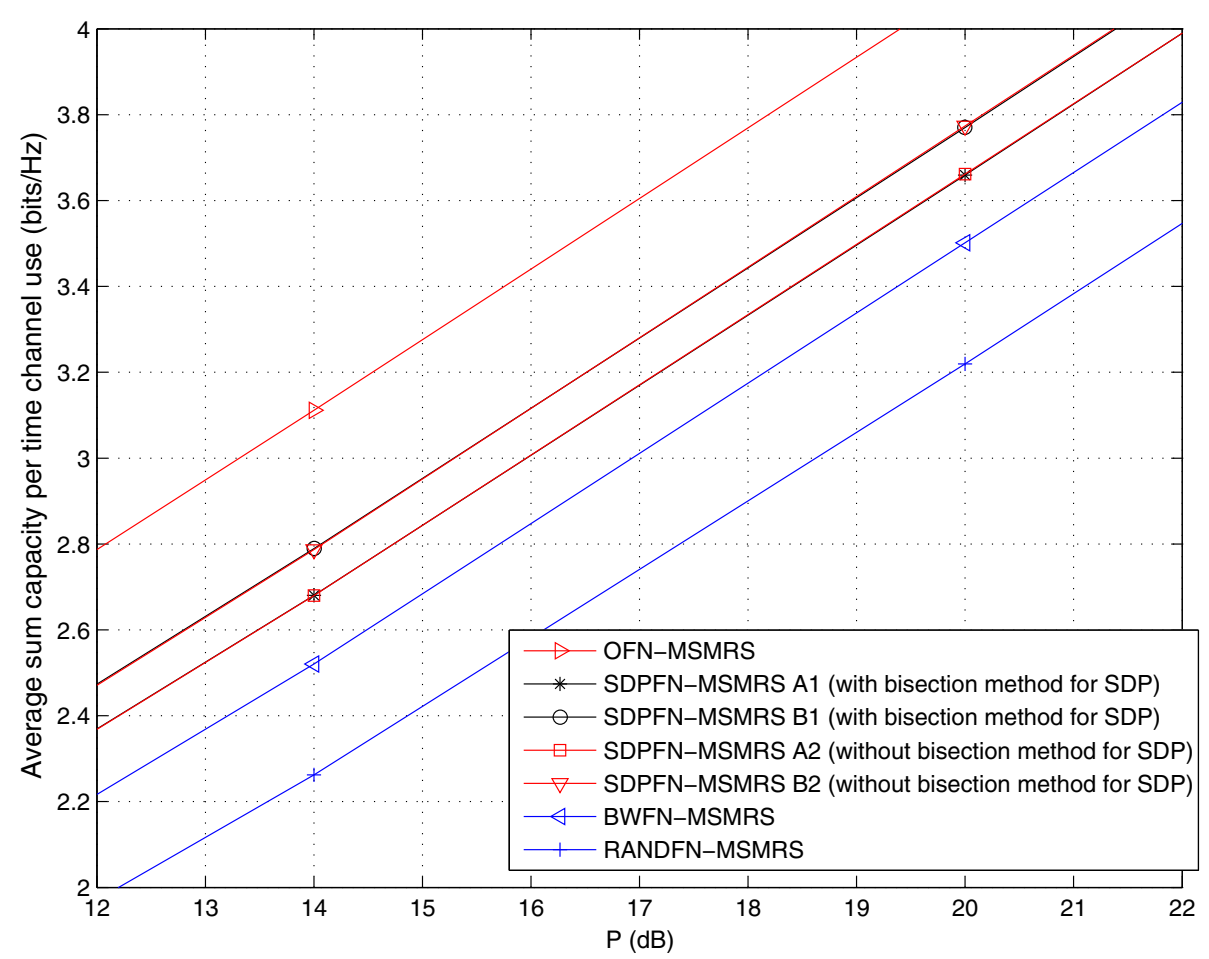

Figure 1 Average per time channel use sum capacity versus $P$ for FN-MSMRS, $K=8, M=2, L=4,\left\{P_{k}^{(R)}=M P, k=1, \ldots, K\right\}$, $\left\{P_{m}^{(S)}=P, m=1, \ldots, M\right\}$. 


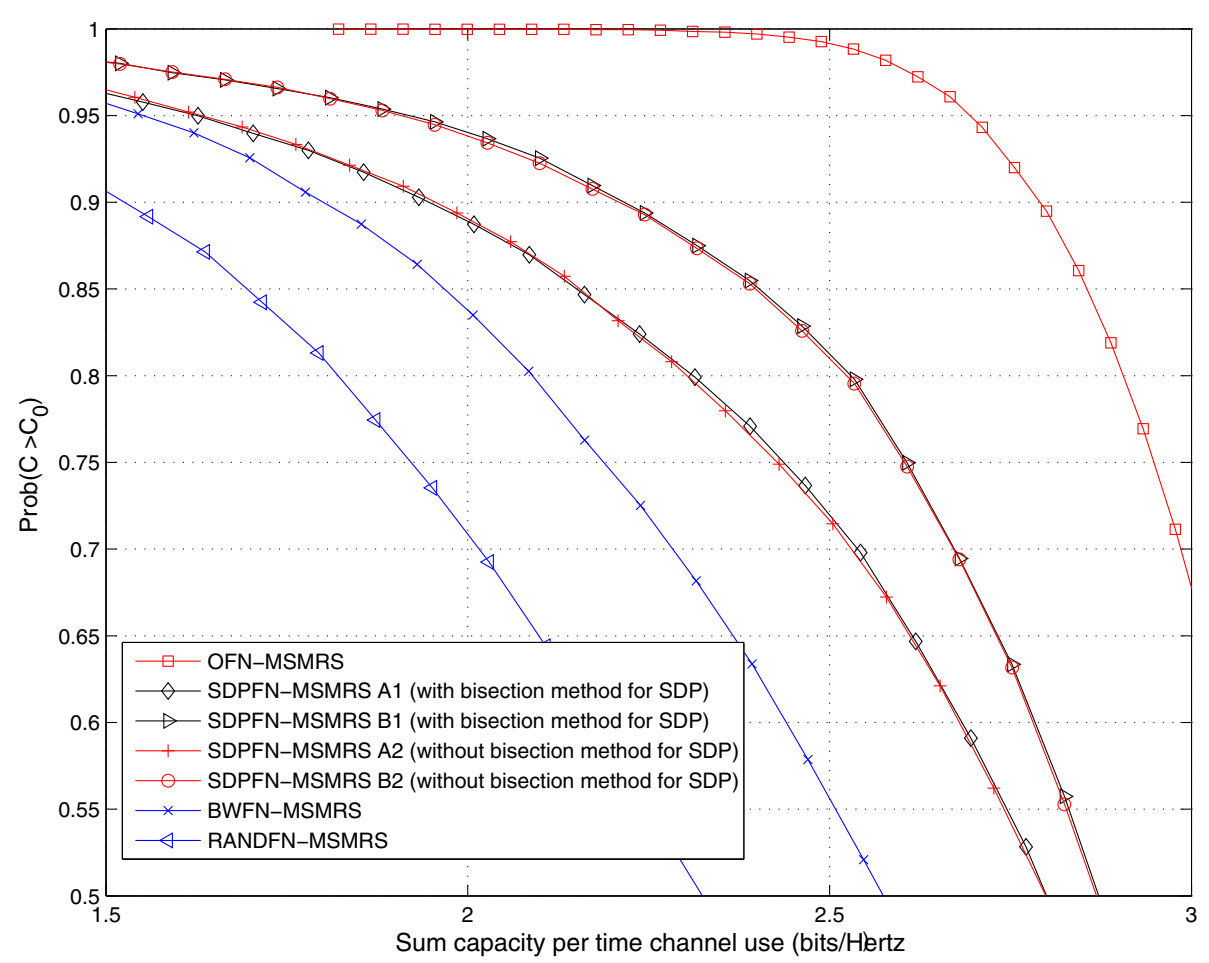

Figure 2 Complementary cumulative distribution function of sum capacity per time channel use at $P=14 \mathrm{~dB}$ for FN-MSMRS, $K=8, M=2, L=4,\left\{P_{k}^{(R)}=M P, k=1, \ldots, K\right\},\left\{P_{m}^{(S)}=P, m=1, \ldots, M\right\}$.

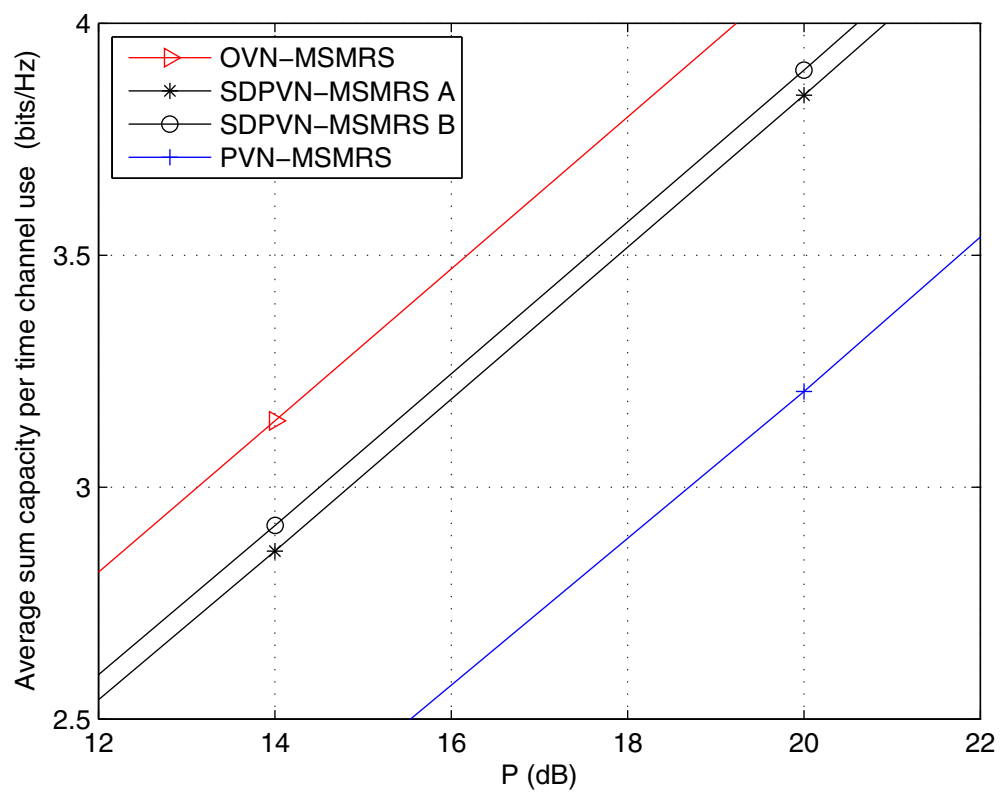

Figure 3 Average per time channel use sum capacity versus $P$ for VN-MSMRS, $K=8, M=2, P^{(\text {Sum })}=4 \mathrm{PM},\left\{P_{k}^{(R)}=\mathrm{PM}, k=1, \ldots, K\right\}$, $\left\{P_{m}^{(S)}=P, m=1, \ldots, M\right\}$. 


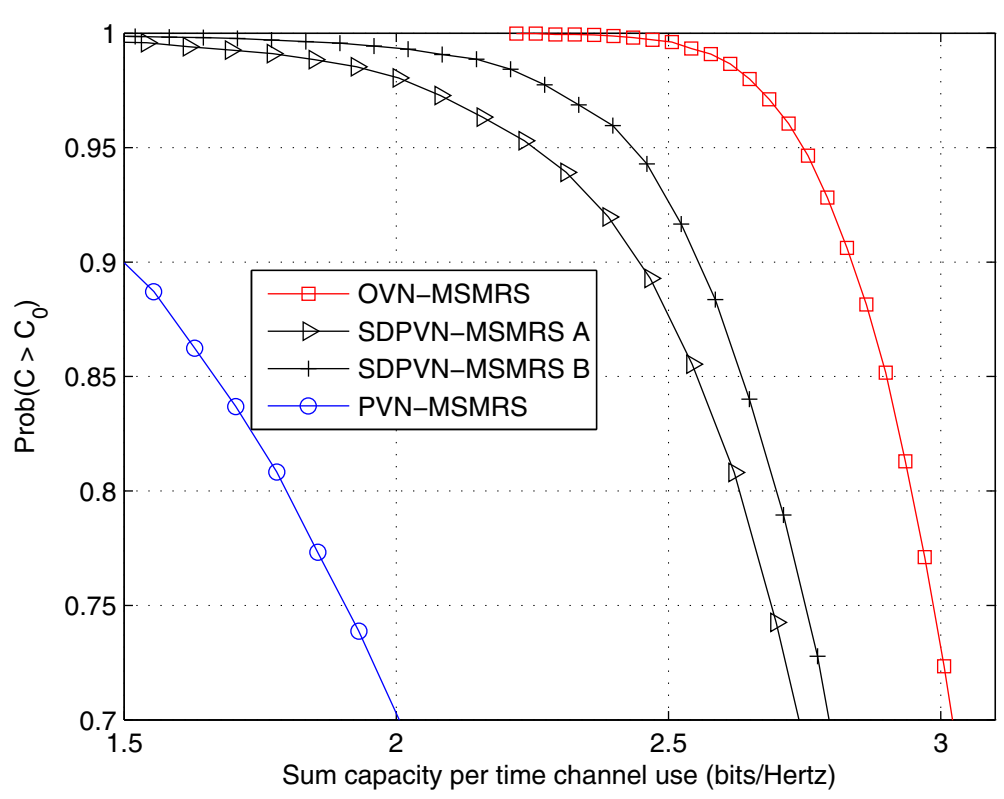

Figure 4 Complementary cumulative distribution function of capacity per time channel use at $\boldsymbol{P}=\mathbf{1 4} \mathrm{dB}$ for VN-MSMRS,

$K=8, M=2, P^{(\mathrm{Sum})}=4 \mathrm{PM},\left\{P_{k}^{(R)}=\mathrm{PM}, k=1, \ldots, K\right\},\left\{P_{m}^{(S)}=P, m=1, \ldots, M\right\}$.

Unlike in Figures 3 and 4, relay powers in Figures 5 and 6 are not uniformly distributed, and we assume $K=$ $8, M=2, P^{(\mathrm{Sum})}=3.62 P M,\left\{P_{k}^{(R)}=P M, k=1,2\right\}$, $\left\{P_{k}^{(R)}=0.65 P M, k=3,4\right\},\left\{P_{k}^{(R)}=0.4 P M, k=5, \ldots, 8\right\}$,
$\left\{P_{m}^{(S)}=P, m=1, \ldots, M\right\}$. In Figure 5 , similar conclusions can be drawn except for different gains as shown in Figure 3. For example, in Figure 5, SDPVN-MSMRS B uses $3.55 \mathrm{~dB}$ less unit power $P$ than PVN-MSMRS. The

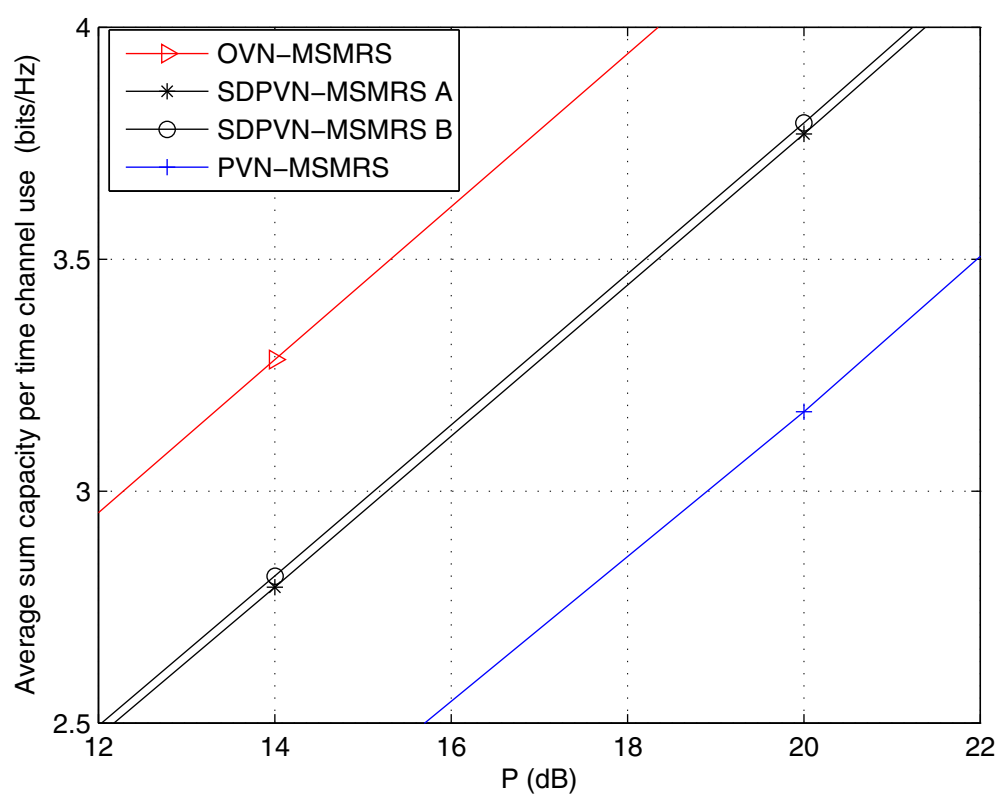

Figure 5 Average per time channel use sum capacity versus $P$ for VN-MSMRS, $K=8, M=2, P^{(\text {sum })}=3.62 \mathrm{PM},\left\{P_{k}^{(R)}=\mathrm{PM}, k=1,2\right\}$, $\left\{P_{k}^{(R)}=0.65 \mathrm{PM}, k=3,4\right\},\left\{P_{k}^{(R)}=0.4 \mathrm{PM}, k=5, \ldots, 8\right\},\left\{P_{m}^{(S)}=P, m=1, \ldots, M\right\}$. 


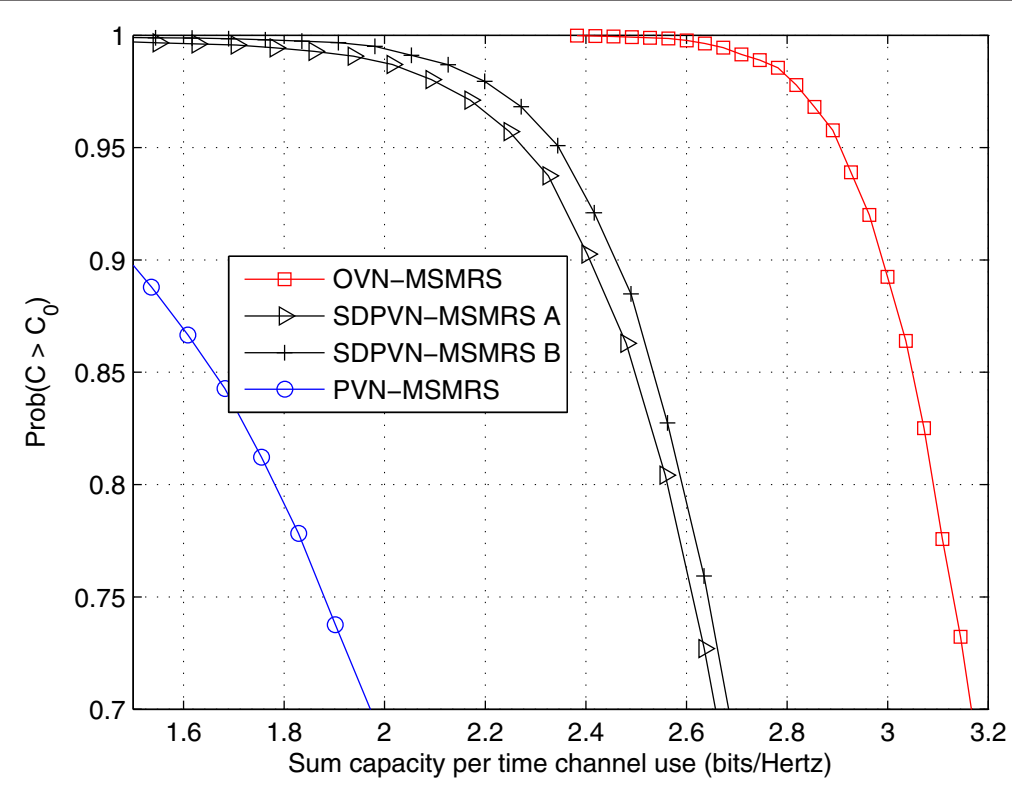

Figure 6 Complementary cumulative distribution function of sum capacity per time channel use at $P=14 \mathrm{~dB}$ for VN-MSMRS,

$K=8, M=2, P^{(\mathrm{Sum})}=3.62 \mathrm{PM},\left\{P_{k}^{(R)}=\mathrm{PM}, k=1,2\right\},\left\{P_{k}^{(R)}=0.65 \mathrm{PM}, k=3,4\right\},\left\{P_{k}^{(R)}=0.4 \mathrm{PM}, k=5, \ldots, 8\right\}$,

$\left\{P_{m}^{(S)}=P, m=1, \ldots, M\right\}$.

above results verify the importance of steps 9 and 10 of Algorithm 3.

\section{Comparison between OFN-MSMRS and OVN-MSMRS}

In Figures 7 and 8, we compare OFN-MSMRS with OVN-MSMRS under the same power constraints, and we assume $K=8, M=2, P^{(\text {Sum })}=4 P M$, $\left\{P_{k}^{(R)}=P M, k=1, \ldots, K\right\}, \quad\left\{P_{m}^{(S)}=P, m=1, \ldots, M\right\}$. Note that, for OFN-MSMRS, $P^{(\text {Sum })}=4 P M$ is equivalent to set $L=4$. It is evident that OVN-MSMRS outperforms OFN-MSMRS under the same power constraints. This

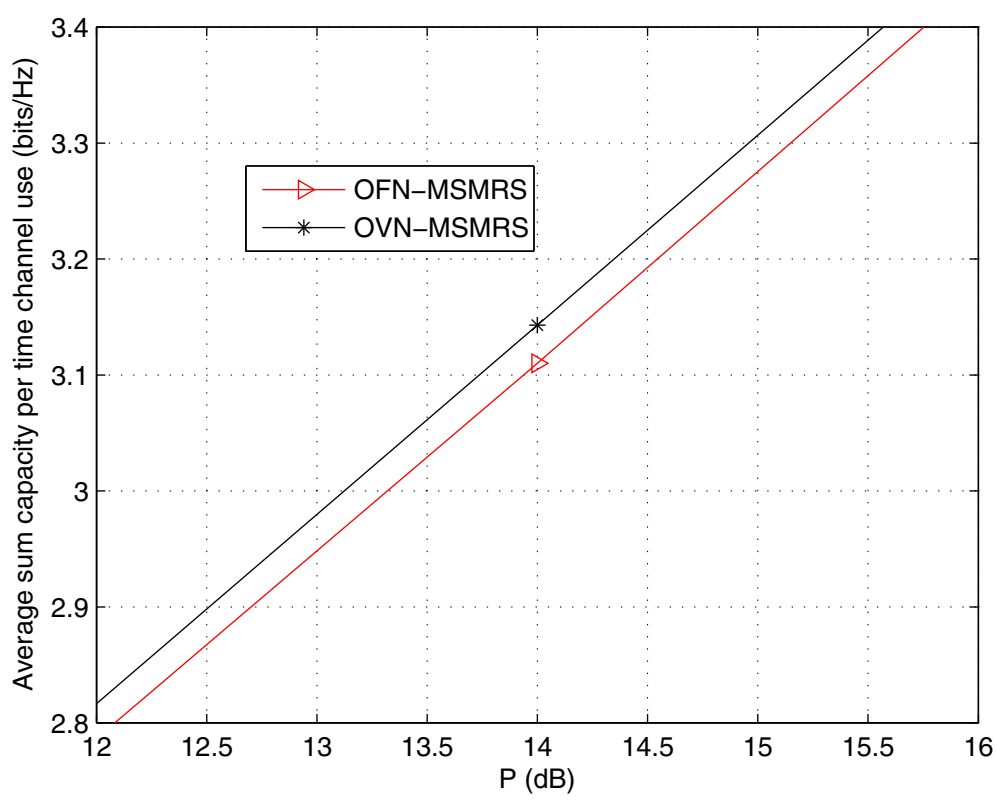

Figure 7 Average per time channel use sum capacity versus $P$ for OFN-MSMRS and OVN-MSMRS, $K=8, M=2, P^{(\text {Sum })}=4$ PM, $\left\{P_{k}^{(R)}=\mathrm{PM}, k=1, \ldots, K\right\},\left\{P_{m}^{(S)}=P, m=1, \ldots, M\right\}$. 


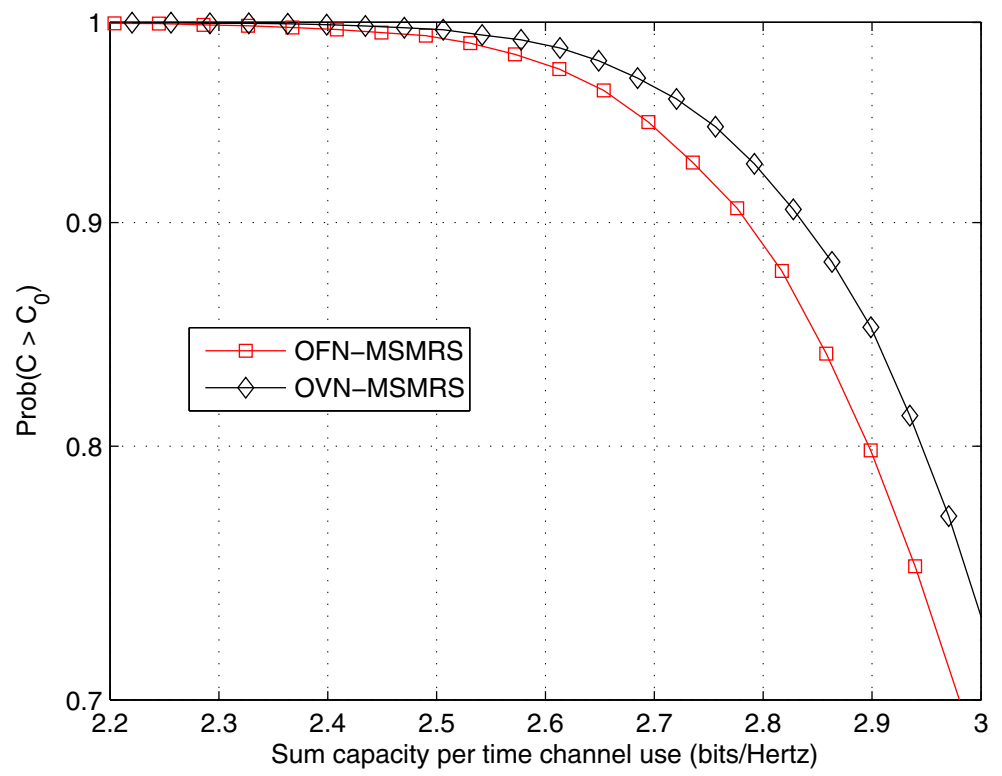

Figure 8 Complementary cumulative distribution function of sum capacity per time channel use at $P=14 \mathrm{~dB}$ for OFN-MSMRS and OVN-MSMRS, $K=8, M=2, P^{(\mathrm{Sum})}=4 \mathrm{PM},\left\{P_{k}^{(R)}=\mathrm{PM}, k=1, \ldots, K\right\},\left\{P_{m}^{(S)}=P, m=1, \ldots, M\right\}$.

implies that the best selection solution for some channel realizations may not necessarily always reach full sum power constraints.

Note that the complexity of optimal MSMRS significantly increases when $K$ becomes larger. In simulations, we choose a small number of $K=8$, for reduced simulation time. For such low $K$ values, the complexity advantage for the proposed approaches may not be that significant. However, with the increase of $K$, complexity advantage for proposed approaches in Sections 'Fixed number multiple-source multiple-relay selection' and 'Varied number multiple-source multiple-relay selection' will become more pronounced.

\section{Conclusion}

Based on the sum capacity maximization criterion, we have proposed a number of multiple-relay selection approaches for simultaneously transmitting multiple source nodes with fixed power relays in an amplifyand-forward cooperative relay network. We propose computationally efficient algorithms based on semidefinite programming for MSMRS with both fixed number and varied number of relays. We have demonstrated that optimal varied number MSMRS outperforms fixed number MSMRS under the same sum power constraints. Although we have discussed the convex relaxation approaches in this article, as the future research directions, it may be deserved to investigate other nonconvex-relaxation approaches with better performance, such as in [52-54].

\section{Endnote}

${ }^{a}$ In [45], the authors express sign operation using notation " $\sigma$ " instead of "sign"

\section{Appendix}

Algorithms

\section{Algorithm 1 Bisection procedure:}

1. Initialize the upper and lower limits of $u, u^{(U)}$ and $u^{(L)}$

2. If $\left(\left|u^{(U)}-u^{(L)}\right|<\varepsilon\right)$, go to step 7), otherwise go to step 3$)$;

3. $u:=\frac{1}{2}\left(u^{(U)}+u^{(L)}\right)$;

4. Perform the SDP optimization procedure for problem (20);

5. If the optimization problem (20) is infeasible or unbounded, $\left\{u^{(U)}:=u\right.$; $\}$

else \{

$$
\begin{aligned}
& u^{(L)}:=u ; \\
& \widehat{\mathbf{B}}=\mathbf{B} ; \\
& \}
\end{aligned}
$$

6. Go to step 2);

7. The optimization procedure ends.

\section{Algorithm 2 Randomization procedure for FN-MSMRS:}

1. Compute $\mathbf{V}$ such that $\widehat{\mathbf{B}}=\mathbf{V}^{\mathcal{T}} \mathbf{V}$, where $\mathbf{V}=\left[\mathbf{v}_{1}, \ldots, \mathbf{v}_{K}\right]$

2. Set $a_{c}=0, a_{s}=0$, and $\rho^{(\max )}=0$;

3. If $a_{s} \neq 0$, go to step 13 ), otherwise go to step 4 );

4. If $a_{c} \geq N_{c}$, \{ 
choose $\widehat{\widehat{\mathbf{c}}}$ using BWFN-MSMRS such that $L$ entries of $\widehat{\widehat{\mathbf{c}}}$ equal to 1 and the rest equal to -1 , then go to step 13);

\} else $\{$ go to step 5); $\}$

5. Set $a_{l}=0$;

6. Choose random vector $\mathbf{u}$ from the uniform distribution on the unit sphere;

7. Compute $\mathbf{c}=\operatorname{sign}\left(\mathbf{V}^{\mathcal{T}} \mathbf{u}\right)$, and thus obtain $\underline{\mathbf{c}}$ as in (15);

8. Compute $\mathbf{p}=\frac{1}{2}(\underline{\mathbf{c}}+1)$;

9. If $\left(\sum_{k=1}^{K}[\mathbf{p}]_{1, k}\right)==L,\{$

Compute $\rho$ based on (18b);

If $\rho>\rho^{(\max )}, \rho^{(\max )}=\rho, \widehat{\widehat{\mathbf{c}}}=\underline{\mathbf{c}}$;

$a_{s}=1$;

\}

10. If $\left(K-\left(\sum_{k=1}^{K}[\mathbf{p}]_{1, k}\right)\right)==L,\{$

Compute $\mathbf{c}=-\mathbf{c},[\mathbf{c}]_{1,1}=1$, and thus obtain $\underline{\mathbf{c}}$;

Compute $\rho$ based on (18b);

If $\rho>\rho^{(\max )}, \rho^{(\max )}=\rho, \widehat{\widehat{\mathbf{c}}}=\underline{\mathbf{c}}$;

$a_{s}=1$;

\}

11. $a_{l}=a_{l}+1$;

12. If $a_{l} \geq N_{l},\{$

$a_{c}=a_{c}+1$

go to 3 );

\}

else $\{$ go to 6$) ;\}$

13. The randomization procedure ends.

\section{Algorithm 3 Randomization procedure for VN-MSMRS:}

1. Compute $\mathbf{V}$ such that $\widehat{\mathbf{B}}=\mathbf{V}^{\mathcal{T}} \mathbf{V}$, where $\mathbf{V}=\left[\mathbf{v}_{1}, \ldots, \mathbf{v}_{K}\right], \mathbf{v}_{k}$ is the $k$ th column vector of $\mathbf{V}$;

2. Set $a_{c}=0, a_{s}=0$, and $\rho^{(\max )}=0$;

3. If $a_{s} \neq 0$, go to step 13), otherwise go to step 4);

4. If $a_{c} \geq N_{c}$, \{

Choose $\widehat{\widehat{\mathbf{c}}}$ using optimal multiple-source single-relay selection such that the sum power constraint (28) is satisfied, then go to step 13);

\} else $\{$ go to step 5); $\}$

5. Set $a_{l}=0$;

6. Choose random vector $\mathbf{u}$ from the uniform distribution on the unit sphere;

7. Compute $\mathbf{c}=\operatorname{sign}\left(\mathbf{V}^{\mathcal{T}} \mathbf{u}\right)$, and thus obtain $\underline{\mathbf{c}}$;

8. If the sum power constraint (28) is satisfied, \{ Compute $\rho$ based on (18b);

If $\rho>\rho^{(\max )}, \rho^{(\max )}=\rho, \widehat{\widehat{\mathbf{c}}}=\underline{\mathbf{c}}$;

$a_{s}=1$;

\}
9. Compute $\mathbf{c}=-\mathbf{c},[\mathbf{c}]_{1,1}=1$, and thus obtain $\underline{\mathbf{c}}$;

10. If the sum power constraint (28) is satisfied, \{ Using c, compute $\rho$ based on (18b);

$$
\text { If } \rho>\rho^{(\max )}, \rho^{(\max )}=\rho, \widehat{\widehat{\mathbf{c}}}=\underline{\mathbf{c}} \text {; }
$$

$a_{s}=1$;

\}

11. $a_{l}=a_{l}+1$

12. if $a_{l} \geq N_{l},\{$

$a_{c}=a_{c}+1$

go to 3 );

\}

else \{ go to 6); $\}$

13. The randomization procedure ends.

\section{Competing interest}

The authors declare that they have no competing interests

\section{Acknowledgements}

The study was performed when J. Wu was with the Center for Advanced Communications, Villanova University, Villanova, PA 19085, USA

\section{Author details}

${ }^{1}$ Bell Laboratories, Alcatel-Lucent, Shanghai 201206, P.R. China. ${ }^{2}$ Center for Advanced Communications, Villanova University, Villanova, PA 19085, USA.

${ }^{3}$ Faculty of Engineering, Ozyegin University, 34794, Istanbul, Turkey.

Received: 28 June 2011 Accepted: 3 July 2012

Published: 16 August 2012

\section{References}

1. A Sendonaris, E Erkip, B Aazhang, User cooperation diversity-part I: system description. IEEE Trans. Commun. 51(11), 1927-1938 (2003)

2. JN Laneman, DNC Tse, GW Wornell, Cooperative diversity in wireless networks: efficient protocols and outage behavior. IEEE Trans. Inf. Theory. 50(12), 3062-3080 (2004)

3. PA Anghel, M Kaveh, On the performance of distributed space-time coding systems with one and two non-regenerative relays. IEEE Trans. Wirel. Commun. 5(3), 682-692 (2006)

4. J Laneman, G Wornell, Distributed space-time-coded protocols for exploiting cooperative diversity in wireless networks. IEEE Trans. Inf. Theory. 49(10), 2415-2425 (2003)

5. $Y$ Jing, B Hassibi, Distributed space-time coding in wireless relay networks. IEEE Trans. Wirel. Commun. 5(12), 3524-3536 (2006)

6. A Reznik, SR Kulkarni, S Verdu, Degraded Gaussian multirelay channels: Capacity and optimal power allocation. IEEE Trans. Inf. Theory. 50(12), 3037-3046 (2004)

7. X Tang, Y Hua, Optimal design of non-regenerative MIMO wireless relays. IEEE Trans. Wirel. Commun, 1398-1407 (2007)

8. N Khajehnouri, AH Sayed, Distributed MMSE relay strategies for wireless sensor networks. IEEE Trans. Signal Process. 55, 3336-3348 (2007)

9. $X \mathrm{Li}, \mathrm{Y}$ Zhang, M Amin, Joint optimization of source power allocation and relay beamforming in multiuser cooperative wireless networks. Mobile Netw. Appl. 16(5), 562-575 (2011)

10. V Sreng, H Yanikomeroglu, DD Falconer, Relayer selection strategies in cellular networks with peer-to-peer relaying. in Proc. IEEE Vehicular Tech. Conf. vol. 3 (2003), pp. 1949-1953

11. A Ribeiro, X Cai, GB Giannakis, Symbol error probabilities for general cooperative links. IEEE Trans. Wirel. Commun. 4, 1264-1273 (2005)

12. AK Sadek, Z Han, KJR Liu, A distributed relay-assignment algorithm for cooperative communications in wireless networks. in Proc. IEEE Int. Conf. Commun. (Istanbul, Turkey, 2006), pp. 1592-1597

13. Y Zhao, R Adve, TJ Lim, Symbol error rate of selection amplify-and-forward relay systems. IEEE Commun. Lett. 10,757-759 (2006)

14. Z Lin, E Erkip, A Stefanov, Cooperative regions and partner choice in coded cooperative systems. IEEE Trans. Commun. 54, 1323-1334 (2006) 
15. DS Michalopoulos, GK Karagiannidis, TA Tsiftsis, RK Mallik, An optimized user selection method for cooperative diversity systems. in Proc. IEEE Globecom (San Francisco, CA, USA, 2006)

16. R Madan, NB Mehta, AF Molisch, J Zhang, Energy-efficient cooperative relaying over fading channels with simple relay selection. in Proc. IEEE Globecom (San Francisco, CA, USA, 2006)

17. CK Lo, RWH Jr, S Vishwanath, Hybrid-ARQ in multihop networks with opportunistic relay selection. in Proc. IEEE Int. Conf. Acoustics, Speech, and Signal Process, vol. 10 (Honolulu, Hawaii, USA, 2007), pp. 617-620

18. Y Zhao, R Adve, TJ Lim, Improving amplify-and-forward relay networks: optimal power allocation versus selection. IEEE Trans. Wirel. Commun. 6 3114-3122 (2007)

19. B Chalise, L Vandendorpe, Y Zhang, M Amin, Local CSI based selection beamforming for amplify-and-forward MIMO relay networks. IEEE Trans. Signal Process. 60(5), 2433-2446 (2012)

20. A Stefanov, E Erkip, Cooperative coding for wireless networks. IEEE Trans, Commun. 52, 1470-1476 (2004)

21. J Luo, RS Blum, LJ Cimini, LJ Greenstein, AM Haimovich, Link-failure probabilities for practical cooperative relay networks. in Proc. IEEE Veh. Tech. Conf. Spring, vol. 3 (Stockholm, Sweden, 2005), pp. 1489-1493

22. A Stefanov, E Erkip, Cooperative space-time coding for wireless networks. IEEE Trans. Commun. 53(11), 1804-1809 (2005)

23. A Bletsas, DP Reed, A Lippman, A simple cooperative diversity method based on network path selection. IEEE J. Sel. Areas Commun. 24, 659-672 (2006)

24. Y Li, B Vucetic, Z Chen, J Yuan, An improved relay selection scheme with hybrid relaying protocols. in Proc. Global Telecommun. Conf. (Washington, D.C., USA, 2007), pp. 3704-3708

25. CK Lo, S Vishwanath, RW Heath, Relay subset selection in wireless networks using partial decode-and-forward transmission. in Proc. Vehicular Tech. Conf. Spring (Marina Bay, Singapore, 2008), pp. 2395-2399

26. R Madan, N Mehta, A Molisch, J Zhang, Energy-efficient cooperative relaying over fading channels with simple relay selection. IEEE Trans. Wirel. Commun. 7(8), 3013-3025 (2008)

27. Y Zhang, Y Xu, Y Cai, Relay selection utilizing power control for decode-and-forward wireless relay networks. in Proc. Int. Conf. on Sig. Proc. Commun. Syst. (Gold Coast, Australia, 2008), pp. 1-5

28. DS Michalopoulos, HA Suraweera, GK Karagiannidis, R Schober, Amplify-and-forward relay selection with outdated channel estimates. IEEE Trans. Commun. 60(5), 1278-1290 (2012)

29. I Krikidis, T Charalambous, JS Thompson, Buffer-aided relay selection for cooperative diversity systems without delay constraints. IEEE Trans. Wirel. Commun. 11(5), 1957-1967 (2012)

30. Z Lin, E Erkip, Relay search algorithms for coded cooperative systems. in Proc. IEEE Globecom, vol. 3 (St. Louis, Missouri, USA, 2005)

31. $Y$ Jing, $H$ Jafarkhani, Single and multiple relay selection schemes and their diversity orders. in Proc. IEEE ICC 2008 Workshop on Cooperative Commun.\& Networking (Beijing, China, 2008), pp. 349-353

32. B Hegyi, J Levendovszky, Efficient, distributed, multiple-relay selection procedures for cooperative communications. in Proc. Int. Symposium Wireless Pervasive Computing (Santorini, Greece, 2008), pp. 170-174

33. $Y$ Jing, $H$ Jafarkhani, Single and multiple relay selection schemes and their diversity orders. IEEE Trans. Wirel. Commun. 8(3), 1414-1423 (2009)

34. B Elzbieta, A Raviraj, Selection cooperation in multi-source cooperative networks. IEEE Trans. Wirel. Commun. 7, 118-127 (2008)

35. J Xu, S Zhou, Z Niu, Interference-aware relay selection for multiple source-destination cooperative networks. in Proc. 15th Asia-Pacific Conf. on Commun. (Shanghai, China, 2009), pp. 338-341

36. W Guo, J Liu, L Zheng, Y Liu, G Zhang, Performance analysis of a selection cooperation scheme in multi-source multi-relay networks. in Proc. Int. Conf. on Wireless Commun. and Signal Processing (Nanjing, China, 2010), pp. 1-6

37. L Ding, M Tao, F Yang, W Zhang, Joint scheduling and relay selection in one- and two-way relay networks with buffering. in Proc. IEEE Int. Conf. Commun. (Dresden, Germany, 2009)

38. I Krikidis, Relay selection for two-way relay channels with MABC DF: A diversity perspective. IEEE Trans. Veh. Technol. 59(9), 4620-4628 (2010)

39. Y Li, RHY Louie, B Vucetic, Relay selection with network coding in two-way relay channels. IEEE Trans. Veh. Technol. 59(9), 4489-4499 (2010)
40. S Talwar, Y Jing, S Shahbazpanahi, Joint relay selection and power allocation for two-way relay networks. IEEE Signal Process. Lett. 18(2), 91-94 (2011)

41. L Ozarow, The capacity of the white Gaussian multiple access channe with feedback. IEEE Trans. Inf. Theory. 30(4), 623-629 (1984)

42. B Rimoldi, R Urbanke, A rate-splitting approach to the Gaussian multiple-access channel. IEEE Trans. Inf. Theory. 42(2), 364-375 (1996)

43. V Aggarwal, A Sabharwal, Slotted Gaussian multiple access channel: Stable throughput region and role of side information. EURASIP J. Wirel. Commun. Network. 2008, 1-11 (2008)

44. L Vandenberghe, S Boyd, Semidefinite programming. SIAM Rev. 38 49-95 (1996)

45. WK Ma, TN Davidson, KM Wong, ZQ Luo, PC Ching, Quasi-maximum-likelihood multiuser detection using semi-definite relaxation with application to synchronous CDMA. IEEE Trans. Signa Process. 50, 912-922 (2002)

46. M Grant, S Boyd, CVX: Matlab software for disciplined convex programming, version 1. 21 (2011), http://cvxr.com/cvx/

47. M Grant, S Boyd, Graph implementations for nonsmooth convex programs. in Recent Advances in Learning and Control, Lecture Notes in Control and Information Sciences (Springer-Verlag Limited, 2008), pp. 95-110. http://stanford.edu/ boyd/graph_dcp.html

48. C Helmberg, F Rendl, R Vanderbei, H Wolkowicz, An interior point method for semidefinite programming. SIAM J. Optimiz. 6(2), 342-361 (1996)

49. A Charnes, WW Cooper, Programming with linear fractional functions. Naval Res. Logist. Quaterly. 9, 181-186 (1962)

50. MX Goemans, DP Williamson, Improved approximation algorithms for maximum cut and satisfiability problem using semi-definite programming. J. ACM. 42(6), 1115-1145 (1995)

51. YE Nesterov, Quality of semidefinite relaxation for nonconvex quadratic optimization, Tech. Rep., CORE, Universite Catholique de Louvain, Brussels, Belgium, 1997

52. Y Zhang, G Zheng, C Ji, K Wong, Near-optimal joint antenna selection for amplify and forward relay networks. IEEE Trans. Wirel. Commun. 9(8), 2401-2407 (2010)

53. J Chen, C Wen, Near-optimal relay subset selection for two-way amplify and forward MIMO relaying systems. IEEE Trans. Wirel. Commun. 10 37-42 (2011)

54. H Park, J Chun, A two-stage antenna subset selection scheme for amplify and forward MIMO relay systems. IEEE Signal Process. Lett. 17(11), 953-956 (2010)

\section{doi:10.1186/1687-1499-2012-256}

Cite this article as: Wu et al.: Multiple-relay selection in amplify-and-forward cooperative wireless networks with multiple source nodes. EURASIP Journal on Wireless Communications and Networking 2012 2012:256.

\section{Submit your manuscript to a SpringerOpen ${ }^{\odot}$ journal and benefit from:}

- Convenient online submission

Rigorous peer review

- Immediate publication on acceptance

- Open access: articles freely available online

- High visibility within the field

- Retaining the copyright to your article

Submit your next manuscript at $>$ springeropen.com 